\title{
PLABIC GRAPHS AND ZONOTOPAL TILINGS
}

\author{
PAVEL GALASHIN
}

\begin{abstract}
We say that two sets $S, T \subset\{1,2, \ldots, n\}$ are chord separated if there does not exist a cyclically ordered quadruple $a, b, c, d$ of integers satisfying $a, c \in S-T$ and $b, d \in T-S$. This is a weaker version of Leclerc and Zelevinsky's weak separation. We show that every maximal by inclusion collection of pairwise chord separated sets is also maximal by size. Moreover, we prove that such collections are precisely vertex label collections of fine zonotopal tilings of the three-dimensional cyclic zonotope. In our construction, plabic graphs and square moves appear naturally as horizontal sections of zonotopal tilings and their mutations respectively.
\end{abstract}

\section{INTRODUCTION}

In 1998, Leclerc and Zelevinsky [9] defined the notion of weak separation while studying the $q$-deformation of the coordinate ring of the flag variety. They showed that for two subsets $S$ and $T$ of the set $[n]:=\{1,2, \ldots, n\}$, the corresponding quantum flag minors quasicommute if and only if $S$ and $T$ are weakly separated which is a certain natural combinatorial condition that we recall below. They raised an exciting purity conjecture: every maximal by inclusion collection of pairwise weakly separated subsets of $[n]$ is also maximal by size. This conjecture has been proven independently by Danilov-Karzanov-Koshevoy [5, 6] and by Oh-Postnikov-Speyer [10]. In particular, Oh, Postnikov, and Speyer showed that maximal by inclusion weakly separated collections of $k$-element sets correspond to reduced plabic graphs (see Figure 3 for an example). Plabic graphs have been introduced by Postnikov in [11] where he used them to construct a certain CW decomposition of the totally nonnegative Grassmannian. Since then it was discovered that plabic graphs have important connections to various fields such as scattering amplitudes [1], soliton solutions to the KP equation, and cluster algebras [8, 7]. It was shown earlier by Scott [13, 14] that maximal by

Date: December 30, 2016.

2010 Mathematics Subject Classification. 52C22(primary), 05E99(secondary).

Key words and phrases. Zonotopal tilings, weak separation, purity phenomenon, plabic graphs, higher Bruhat order. 
inclusion weakly separated collections of subsets form clusters in the cluster algebra structure on the coordinate ring of the Grassmannian.

Let us recall the definition of weak separation from [9]. Given two subsets $S, T$ of $[n]$, we say that $S$ surrounds $T$ if there do not exist numbers $i<j<k \in[n]$ such that $i, k \in T-S$ and $j \in S-T$. We say that $S$ and $T$ are strongly separated if $S$ surrounds $T$ and $T$ surrounds $S$. We say that $S$ and $T$ are weakly separated if at least one of the following holds:

(1) $|S| \leq|T|$ and $S$ surrounds $T$;

(2) $|T| \leq|S|$ and $T$ surrounds $S$.

This definition simplifies considerably when $|S|=|T|$. Namely, let us say that $S$ and $T$ are chord separated if there do not exist numbers $a<b<c<d \in[n]$ such that $a, c \in S-T$ and $b, d \in T-S$ or vice versa. In other words, $S$ and $T$ are chord separated if either $S$ surrounds $T$ or $T$ surrounds $S$. One easily observes that when $|S|=|T|$, the two sets are weakly separated if and only if they are chord separated. However, for general $S$ and $T$ our notion of chord separation is weaker than that of weak separation.

We say that a collection $\mathcal{D} \subset 2^{[n]}$ of subsets of $[n]$ is weakly separated if any two sets $S, T \in \mathcal{D}$ are weakly separated from each other. We can now state the purity phenomenon for weak separation:

Theorem 1.1 (see [10, [5, 6]). (1) Every maximal by inclusion weakly separated collection $\mathcal{D} \subset 2^{[n]}$ has size

$$
\left(\begin{array}{l}
n \\
2
\end{array}\right)+\left(\begin{array}{l}
n \\
1
\end{array}\right)+\left(\begin{array}{l}
n \\
0
\end{array}\right)
$$

(2) Every maximal by inclusion weakly separated collection $\mathcal{D} \subset$ $\left(\begin{array}{c}{[n]} \\ k\end{array}\right)$ has size

$$
k(n-k)+1 .
$$

Here $\left(\begin{array}{c}{[n]} \\ k\end{array}\right)$ denotes the collection of all k-element subsets of $[n]$.

The second statement shows that every maximal by inclusion chord separated collection $\mathcal{D} \subset\left(\begin{array}{c}{[n]} \\ k\end{array}\right)$ of $k$-element sets is also maximal by size. As we have already mentioned, Oh-Postnikov-Speyer showed that such collections are in a one-to-one correspondence with reduced plabic graphs (see Section 2.3 for the definition).

The second part of our story involves zonotopal tilings. Zonotopes are Minkowski sums of segments, and zonotopal tilings of a given zonotope $\mathcal{Z}$ are polytopal subdivisions of $\mathcal{Z}$ into smaller zonotopes. We say that a zonotopal tiling is fine (also called tight in the literature) if all of its zonotopes are parallelotopes. Zonotopal tilings have been a 


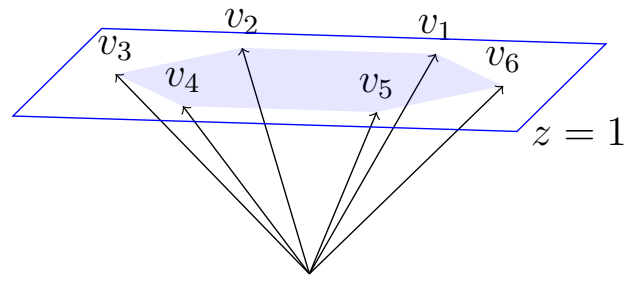

Figure 1. The configuration $\mathbf{C}(6,3)$.

popular subject of research for a long time, and have received much attention during the past two decades in the context of the generalized Baues problem, see [2], [12], and [3, Section 7.2]. Another famous conjecture, the Extension space conjecture, is related to zonotopal tilings via the celebrated Bohne-Dress theorem [4] which gives an unexpected correspondence between zonotopal tilings of a given zonotope and oneelement liftings of the associated oriented matroid. We are going to be interested in the three-dimensional cyclic zonotope $\mathcal{Z}(n, 3)$ that is associated to the cyclic vector configuration $\mathbf{C}(n, 3)$. The endpoints of vectors in $\mathbf{C}(n, 3)$ are the vertices of a convex $n$-gon lying in the affine plane $z=1$, see Figure 1. Given a fine zonotopal tiling $\Delta$ of $\mathcal{Z}(n, 3)$, its vertices are naturally labeled by subsets of $[n]$ (see Figure 2), and we denote this collection of subsets by $\operatorname{Vert}(\Delta) \subset 2^{[n]}$.

We are now ready to state our main result.

Theorem 1.2. • Any maximal by inclusion chord separated collection $\mathcal{D} \subset 2^{[n]}$ has size

$$
\left(\begin{array}{l}
n \\
0
\end{array}\right)+\left(\begin{array}{l}
n \\
1
\end{array}\right)+\left(\begin{array}{l}
n \\
2
\end{array}\right)+\left(\begin{array}{l}
n \\
3
\end{array}\right) .
$$

- Given any integer $0 \leq k \leq n$, the collection $\mathcal{D} \cap\left(\begin{array}{c}{[n]} \\ k\end{array}\right)$ has size $k(n-k)+1$, that is, is maximal by size in $\left(\begin{array}{c}{[n]} \\ k\end{array}\right)$ and thus corresponds to a plabic tiling, which we denote $\Sigma_{k}(\mathcal{D})$.

- The map $\Delta \mapsto \operatorname{Vert}(\Delta)$ gives a bijection between fine zonotopal tilings of $\mathcal{Z}(n, 3)$ and maximal by inclusion chord separated collections $\mathcal{D} \subset 2^{[n]}$. For any $0 \leq k \leq n$, the intersection of $\Delta$ with the plane $z=k$ in $\mathbb{R}^{3}$ gives a triangulation of $\Sigma_{k}(\mathcal{D})$ where $\mathcal{D}=\operatorname{Vert}(\Delta)$ is the corresponding chord separated collection.

The conclusion is, triangulated plabic tilings (or dually, reduced trivalent plabic graphs) are sections of fine zonotopal tilings of the cyclic zonotope $\mathcal{Z}(n, 3)$. In fact, we give a simple compatibility condition on when two triangulated plabic tilings $\Sigma_{k}$ and $\Sigma_{k+1}$ can appear as the corresponding sections of a fine zonotopal tiling, see Definition 4.3. 


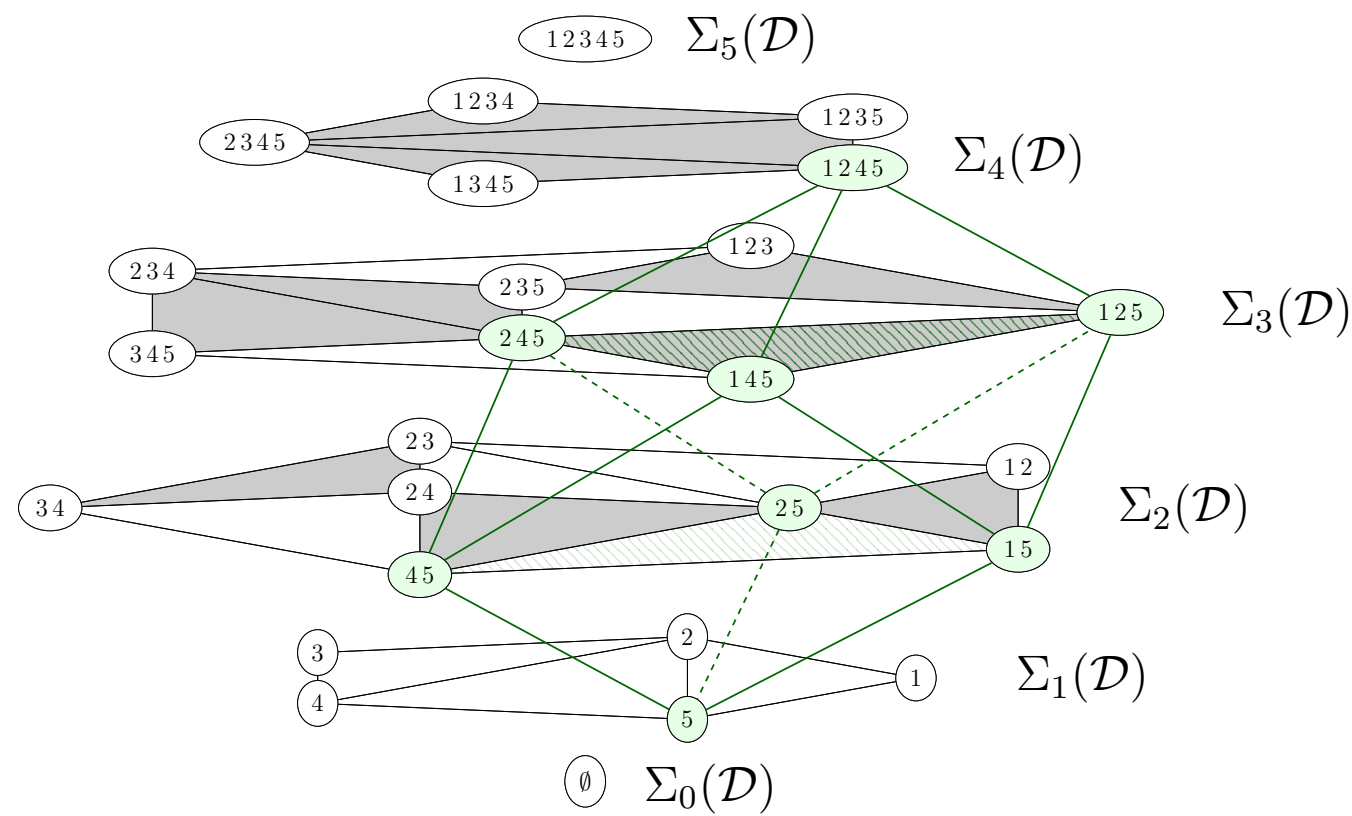

Figure 2. A maximal by inclusion chord separated collection $\mathcal{D}$ of subsets of $\{1,2,3,4,5\}$ and the corresponding zonotopal tiling $\Delta(\mathcal{D})$ of $\mathcal{Z}(5,3)$. One of the 10 tiles is shown in green. Each horizontal section $\Sigma_{i}(\mathcal{D})$, $i=0,1, \ldots, 5$, is a triangulation of a plabic tiling.

Theorem 1.2 is illustrated in Figure 2. Namely, the size of the collection equals $26=1+5+10+10$, the sections are clearly triangulated plabic tilings, and one can easily observe that these sections together form a fine zonotopal tiling $\Delta$ : the eight vertices of any zonotope in $\Delta$ are of the form $S, S a, S b, S c, S a b, S a c, S b c, S a b c \in \mathcal{D}$. Here by $S a$ we abbreviate $S \cup\{a\}$, etc. The vertices $S a, S b, S c$ form a white triangle in the plabic tiling $\Sigma_{|S|+1}(\mathcal{D})$ and the vertices $S a b, S b c, S a c$ form a black triangle in $\Sigma_{|S|+2}(\mathcal{D})$. For example, $\{2,23,12,25,123,235,125,1235\}$ are the vertices of one of the 10 tiles of $\Delta$. This tile is highlighted in green in Figure 2 .

Remark 1.3. In [9, Theorem 1.6], Leclerc and Zelevinsky showed that all maximal by inclusion strongly separated collections have size

$$
\left(\begin{array}{l}
n \\
0
\end{array}\right)+\left(\begin{array}{l}
n \\
1
\end{array}\right)+\left(\begin{array}{l}
n \\
2
\end{array}\right)
$$

and correspond to pseudoline arrangements, or dually, to zonotopal tilings of the two-dimensional cyclic zonotope. Thus our Theorem 1.2 is a direct three-dimensional analog of their result. 
In Section 2, we introduce necessary notation and review the definitions for zonotopal tilings and plabic graphs. We then discuss how plabic graphs, square moves, and strands appear as horizontal sections of their three-dimensional counterparts in Section 3. Finally, we prove Theorem 1.2 in Section 4.

\section{ACKNOWLEDGMENT}

The author is grateful to Alex Postnikov for his numerous useful suggestions and ideas.

\section{BACKGROUND}

Given a set $S$ and an element $e \notin S$, we denote by $S e$ the union of $S$ and $\{e\}$. The use of $S e$ indicates that $e \notin S$. By $S-T$ we denote the set-theoretic difference of sets $S$ and $T$, in particular, $S-e=S-\{e\}$. This notation does not imply that $T \subset S$ or $e \in S$. For $a, b \in[n]$, by $[a, b]$ we denote the cyclic interval $\{a, a+1, \ldots, b-1, b\}$. The indices are always taken modulo $n$, so if $a>b$ then $[a, b]=[a, n] \cup[1, b]$. Similarly, $(a, b)$ denotes $[a+1, b-1]$ and $(a, b]$ denotes $[a+1, b]$, etc.

2.1. Zonotopal tilings. A signed subset $X$ of $[n]$ is a pair $\left(X^{+}, X^{-}\right)$of disjoint subsets of $[n]$. In this case we define its support $\underline{X}:=X^{+} \sqcup X^{-}$ and $X^{0}:=[n]-\underline{X}$. We denote the set of all signed subsets of $[n]$ by $\{+,-, 0\}^{[n]}$ and for each element $i \in[n]$ we write

$$
X_{i}= \begin{cases}+, & \text { if } i \in X^{+} \\ -, & \text {if } i \in X^{-} \\ 0, & \text { if } i \in X^{0}\end{cases}
$$

A vector configuration $\mathbf{V}=\left(\mathbf{v}_{1}, \ldots, \mathbf{v}_{n}\right)$ is a finite subset of $\mathbb{R}^{d}$.

For a vector configuration $\mathbf{V}$, define the corresponding zonotope $\mathcal{Z}_{\mathbf{V}}$ to be the Minkowski sum

$$
\mathcal{Z}_{\mathbf{V}}:=\left[0, \mathbf{v}_{1}\right]+\left[0, \mathbf{v}_{2}\right]+\cdots+\left[0, \mathbf{v}_{n}\right],
$$

where the Minkowski sum of two subsets $A, B \subset \mathbb{R}^{d}$ is defined by

$$
A+B=\{a+b \mid a \in A, b \in B\} \subset \mathbb{R}^{d} .
$$

For a signed set $X$, we denote by $\tau_{X}$ the following zonotope:

$$
\tau_{X}:=\sum_{i \in[n]} \begin{cases}v_{i}, & \text { if } i \in X^{+} \\ 0, & \text { if } i \in X^{-} \\ {\left[0, v_{i}\right],} & \text { if } i \in X^{0}\end{cases}
$$


Definition 2.1. A collection $\Delta$ of signed subsets of $[n]$ is called a zonotopal tiling of $\mathcal{Z}_{\mathbf{V}}$ if and only if the following two conditions hold:

- $\mathcal{Z}_{\mathrm{V}}=\bigcup_{X \in \Delta} \tau_{X}$

- For any two $X, Y \in \Delta$, either the intersection $\tau_{X} \cap \tau_{Y}$ is empty or there exists $Z \in \Delta$ such that $\tau_{Z}$ is a face of $\tau_{X}$ and of $\tau_{Y}$, and

$$
\tau_{X} \cap \tau_{Y}=\tau_{Z}
$$

A zonotopal tiling $\Delta$ is called fine if for every $X \in \Delta,\left|X^{0}\right| \leq d$, that is, if all the top-dimensional tiles are parallelotopes. For a fine zonotopal tiling $\Delta$, its set of vertices is defined as

$$
\operatorname{Vert}(\Delta):=\left\{X^{+} \mid X \in \Delta \text { such that } \underline{X}=[n]\right\} \subset 2^{[n]} \text {. }
$$

For $1 \leq d \leq n$, the cyclic vector configuration $\mathbf{C}(n, d)$ is given by the following collection of vectors $\left\{\mathbf{v}_{1}, \ldots, \mathbf{v}_{n}\right\} \subset \mathbb{R}^{d}$, where

$$
\mathbf{v}_{i}=\left(1, x_{i}, \ldots, x_{i}^{d-1}\right)
$$

and $0<x_{1}<x_{2}<\cdots<x_{n} \in \mathbb{R}$ are any increasing positive real numbers.

2.2. Plabic graphs. A planar bicolored graph (plabic graph for short) $G$ is a planar graph embedded in a disc so that every non-boundary vertex of $G$ is colored either black or white. Two adjacent vertices are not required to have opposite colors. Plabic graphs were introduced by Postnikov in [11] in his study of the totally nonnegative Grassmannian.

Given a plabic graph $G$, a strand in $G$ is a path $\left(v_{1}, v_{2}, \ldots, v_{r}\right)$ that "makes a sharp right turn" at every black vertex and "makes a sharp left turn" at every white vertex. More precisely, for any $1<i<r$, the vertices $v_{i-1}, v_{i}, v_{i+1}$ belong to the same face $F$ of $G$ and if $v_{i}$ is white (resp., black) then $v_{i-1}, v_{i}, v_{i+1}$ go in the counterclockwise (resp., clockwise) direction in the boundary of $F$. Let $b_{1}, \ldots, b_{n}$ be the boundary vertices of $G$ in a counterclockwise order. Then the strand permutation $\pi_{G}$ of $[n]$ is defined by $\pi_{G}(i)=j$ whenever the strand that starts at $b_{i}$ ends at $b_{j}$. We denote such a strand by $i \rightarrow j$. See Figure 3 ,

We say that two strands have an essential intersection if there is an edge that they traverse in opposite directions. We say that two strands have a bad double crossing if they have essential intersections at two edges $e_{1}$ and $e_{2}$ and each of the strands first passes through $e_{1}$ and then through $e_{2}$.

Definition 2.2. A plabic graph $G$ is called reduced (see [11, Theorem 13.2]) if

- there are no closed strands in $G$; 


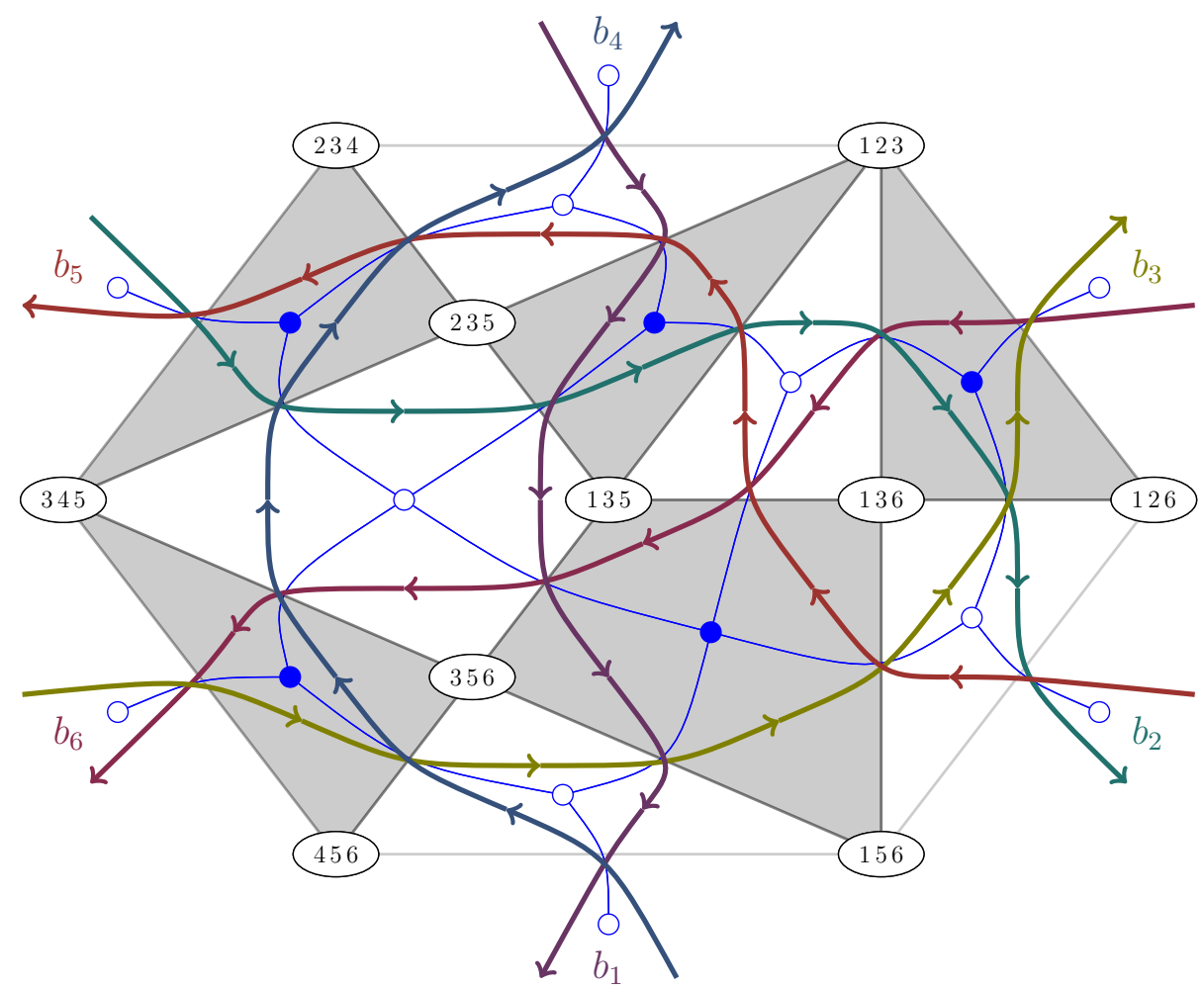

Figure 3. A plabic graph $G$ (blue). For each $j \in[n]$, the faces whose labels contain $j$ are to the left of the strand $i \rightarrow j$. The collection of face labels is a maximal by inclusion weakly separated collection in $\left(\begin{array}{c}{[n]} \\ k\end{array}\right)$. The dual of $G$ is a plabic tiling from Section 2.3 ,

- no strand in $G$ has an essential self-intersection;

- no two strands in $G$ have a bad double crossing;

- if $\pi_{G}(i)=i$ then $G$ has a boundary leaf attached to $b_{i}$.

It follows from this definition that a plabic graph cannot have loops or parallel edges. We will additionally assume that it has no nonboundary vertices of degree two.

We will from now on restrict our attention to reduced plabic graphs whose strand permutation sends $i$ to $i+k$ modulo $n$, for all $i \in[n]$. We denote this permutation by $\sigma^{(k, n)}$. In the stratification of the totally nonnegative Grassmannian from [11, $\sigma^{(k, n)}$ corresponds to the topdimensional cell of $\mathrm{Gr}_{k, n}^{\mathrm{tnn}}$. 


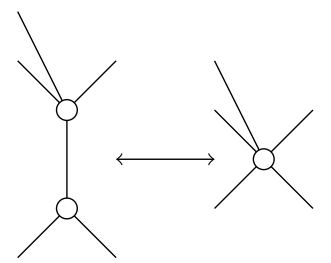

A contraction/uncontraction move

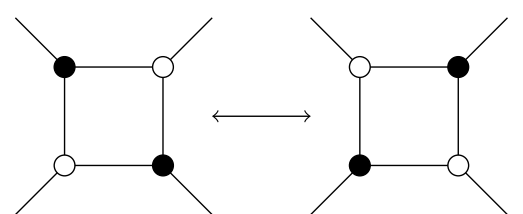

A square move (M2)

Figure 4. Two types of moves on plabic graphs

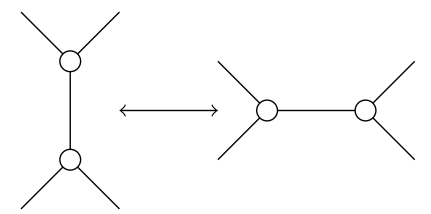

A white trivalent move (M1)

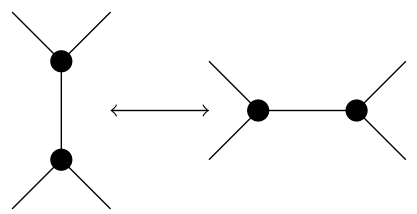

A black trivalent move (M3)

FIGURE 5. Trivalent contraction-uncontraction moves

Remark 2.3. According to our Theorem 1.2 combined with the results of [10], reduced plabic graphs are precisely the objects dual to horizontal sections of fine zonotopal tilings of $\mathcal{Z}(n, 3)$. It is quite surprising that all the conditions from Definition 2.2 are somehow incorporated in the concept of a zonotopal tiling.

As it was shown in [11], all reduced plabic graphs with the same strand permutation are connected by certain moves which we now recall. There are two kinds of moves, unicolored contraction/uncontraction moves and square moves, see Figure 4. Note that in order to perform a square move, all four vertices are required to have degree 3.

Using contraction/uncontraction moves, one can always transform a plabic graph into a trivalent plabic graph for which all non-boundary vertices have degree 3. Any two trivalent plabic graphs are connected by trivalent versions of the moves from Figure 4, namely, by square moves and contraction-uncontraction moves from Figure 5. We denote the square move by (M2) and the white (resp., black) trivalent contraction-uncontraction move by (M1) (resp., (M3)).

Theorem 2.4 ([11]). All reduced plabic graphs with the same strand permutation are connected by moves from Figure 4. All trivalent reduced plabic graphs with the same strand permutation are connected by moves (M1)-(M3).

Given a reduced plabic graph $G$, one can associate to it a certain collection $\mathcal{F}(G) \subset 2^{[n]}$ of face labels of $G$. Namely, for each face $F$ of 
$G$, its label $\lambda(F) \subset[n]$ contains all indices $j \in[n]$ such that $F$ is to the left of the strand $i \rightarrow j$. We set

$$
\mathcal{F}(G)=\{\lambda(F) \mid F \text { is a face of } G\} .
$$

Clearly, if faces $F_{1}$ and $F_{2}$ share an edge then $\lambda\left(F_{1}\right)$ and $\lambda\left(F_{2}\right)$ have the same size, and thus $\mathcal{F}(G)$ only contains sets of the same size.

Theorem 2.5 (see [10]). For any reduced plabic graph $G$ with $\pi_{G}=$ $\sigma^{(k, n)}$, the collection $\mathcal{F}(G)$ is a maximal by inclusion weakly separated collection inside $\left(\begin{array}{c}{[n]} \\ k\end{array}\right)$, and conversely, $\mathcal{D} \subset\left(\begin{array}{c}{[n]} \\ k\end{array}\right)$ is a maximal by inclusion weakly separated collection if and only if there is a reduced plabic graph $G$ with $\pi_{G}=\sigma^{(k, n)}$ and $\mathcal{F}(G)=\mathcal{D}$.

Given that all reduced plabic graphs $G$ with $\pi_{G}=\sigma^{(k, n)}$ are connected by moves from Figure 4 which do not change the cardinality of $\mathcal{F}(G)$, we get that all maximal by inclusion weakly separated collections in $\left(\begin{array}{c}{[n]} \\ k\end{array}\right)$ have the same size, so the purity phenomenon (Theorem [1.1, part 2) follows from Theorems 2.5] and 2.4.

Remark 2.6. The first part of Theorem 1.1 follows from an analogous result in [10] concerning weakly separated collections inside a positroid. Our construction, for the most part, can be repeated for positroids and Grassmann necklaces. However we prefer to just work with plabic graphs satisfying $\pi_{G}=\sigma^{(k, n)}$ for simplicity.

It is quite straightforward to pass from a reduced plabic graph $G$ to its collection $\mathcal{F}(G)$ of face labels. To go in the converse direction, we need the construction of plabic tilings by Oh, Postnikov and Speyer [10] which we review in the next section. Figure 3 shows a plabic graph $G$ with $\pi_{G}=\sigma^{(3,6)}$ together with its face labels and its dual plabic tiling.

2.3. Plabic tilings. Our goal in this section is to assign a certain polygonal subdivision $\Sigma(\mathcal{D})$ of a convex polygon to each maximal by inclusion weakly separated collection $\mathcal{D} \subset\left(\begin{array}{c}{[n]} \\ k\end{array}\right)$ of sets of the same size.

Fix a cyclic vector configuration $\mathbf{C}(n, 3)=\left(\mathbf{v}_{1}, \ldots, \mathbf{v}_{n}\right)$ in $\mathbb{R}^{3}$. For each subset $S \subset[n]$, define the point

$$
\mathbf{v}_{S}:=\sum_{i \in S} \mathbf{v}_{i}
$$

Now, consider a maximal by inclusion chord-separated collection $\mathcal{D} \subset\left(\begin{array}{c}{[n]} \\ k\end{array}\right)$ for some $1 \leq k \leq n$. For each set $K \in\left(\begin{array}{c}{[n]} \\ k-1\end{array}\right)$, define the corresponding white clique

$$
\mathcal{W}(K):=\{S \in \mathcal{D} \mid S \supset K\} .
$$


Similarly, for each set $L \in\left(\begin{array}{c}{[n]} \\ k+1\end{array}\right)$, define the corresponding black clique

$$
\mathcal{B}(L):=\{S \in \mathcal{D} \mid S \subset L\} .
$$

A clique is called non-trivial if it contains at least three elements. Every white clique has a form $\left\{K a_{1}, K a_{2}, \ldots K a_{r}\right\}$ for some $a_{1}<a_{2}<\cdots<$ $a_{r} \in[n]$. Similarly, every black clique has a form $\left\{L-b_{1}, L-b_{2}, \ldots L-\right.$ $\left.b_{s},\right\}$ for some $b_{1}<b_{2}<\cdots<b_{s} \in[n]$. For a non-trivial white clique $\mathcal{W}(K)$, its boundary $\partial \mathcal{W}(K)$ is the following collection of pairs of subsets in $\mathcal{D}$ :

$$
\partial \mathcal{W}(K)=\left\{\left(K a_{1}, K a_{2}\right),\left(K a_{2}, K a_{3}\right), \ldots,\left(K a_{r-1}, K a_{r}\right),\left(K a_{r}, K a_{1}\right)\right\} .
$$

For a non-trivial black clique $\mathcal{B}(L)$, its boundary $\partial \mathcal{B}(L)$ is the following collection of pairs of subsets in $\mathcal{D}$ :

$\partial \mathcal{B}(L)=\left\{\left(L-b_{1}, L-b_{2}\right),\left(L-b_{2}, L-b_{3}\right), \ldots,\left(L-b_{r-1}, L-b_{r}\right),\left(L-b_{r}, L-b_{1}\right)\right\}$.

Oh-Postnikov-Speyer define the following two-dimensional complex $\Sigma(\mathcal{D})$ embedded in $\mathbb{R}^{3}$ :

- The vertices of $\Sigma(\mathcal{D})$ are $\left\{\mathbf{v}_{S} \mid S \in \mathcal{D}\right\}$.

- The edges of $\Sigma(\mathcal{D})$ connect $\mathbf{v}_{S}$ and $\mathbf{v}_{T}$ for every $(S, T)$ belonging to the boundary of a non-trivial clique;

- The two-dimensional faces of $\Sigma(\mathcal{D})$ are polygons whose vertices form a non-trivial clique.

The plabic tiling corresponding to the collection from Figure 3 is depicted in Figure 7 (top). The following result is proven in [10]:

Theorem 2.7 ([10]). If $\mathcal{D} \subset\left(\begin{array}{c}{[n]} \\ k\end{array}\right)$ is a maximal by inclusion chord separated collection of subsets, then it is also maximal by size:

$$
|\mathcal{D}|=k(n-k)+1 \text {. }
$$

Moreover, $\Sigma(\mathcal{D})$ forms a polygonal subdivision of the convex polygon with $n$ vertices labeled by cyclic intervals in $[n]$ of length $k$.

Corollary 2.8 ([10]). Given a reduced plabic graph $G$ with $\pi_{G}=\sigma^{(k, n)}$, the plabic tiling $\Sigma(\mathcal{F}(G))$ is planar dual to $G$.

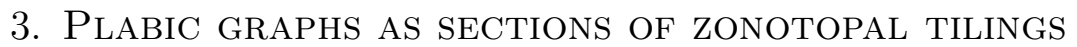

Before we pass to the proof of Theorem 1.2, we would like to discuss informally how to view notions related to plabic graphs as sections of three-dimensional objects related to zonotopal tilings.

Let $\Delta$ be a fine zonotopal tiling of $\mathcal{Z}(n, 3)$. Let $\Delta_{i}$ denote the horizontal sections of $\Delta$ by hyperplanes $H_{i}:=\{(x, y, z) \mid z=i\} \subset \mathbb{R}^{3}$. First, as we have already mentioned in Theorem 1.2, the sections $\Delta_{i}$ 
are triangulations of plabic tilings corresponding to maximal by inclusion weakly separated collections inside $\left(\begin{array}{c}{[n]} \\ k\end{array}\right)$. Conversely, as we will see in the proof of Theorem 1.2 in Section 4, every triangulation of any plabic tiling is a horizontal section of some fine zonotopal tiling of $\mathcal{Z}(n, 3)$. Recall that the dual object to a triangulation of a plabic tiling is a trivalent reduced plabic graph $G$ such that $\pi_{G}=\sigma^{(k, n)}$. Thus we get plabic graphs satisfying $\pi_{G}=\sigma^{(k, n)}$ as duals of horizontal sections of fine zonotopal tilings $\Delta$ of $\mathcal{Z}(n, 3)$.

Next, the objects dual to fine zonotopal tilings of $\mathcal{Z}(n, 3)$ are threedimensional pseudoplane arrangements, which are analogous to the well-studied pseudoline arrangements in two dimensions. Loosely speaking, given $\Delta$ and any $j \in[n]$, we get a pseudoplane $P_{j}$ which is a smooth embedding of $\mathbb{R}^{2}$ (together with an orientation) into $\mathbb{R}^{3}$ that only intersects the tiles $\tau_{X}$ of $\Delta$ satisfying $X_{j}=0$, i.e. $P_{j}$ intersects all segments of $\Delta$ that are parallel to $\mathbf{v}_{j}$. In other words, the collection of vertex labels of $\Delta$ lying on the negative side of $P_{j}$ consists precisely of those elements of $\operatorname{Vert}(\Delta)$ that do not contain $j$.

The formal definition of a pseudoplane arrangement is somewhat more complicated and involves other topological conditions like the intersection of $P_{i}$ and $P_{j}$ being homeomorphic to a line for $i \neq j \in[n]$. We will impose an extra condition that the normal to $P_{j}$ at any point cannot be vertical for all $j \in[n]$.

The reason we are considering pseudoplanes is that their horizontal sections are strands in plabic graphs. Let $G$ be the plabic graph dual to $\Delta_{i}$, and let $\ell=\pi_{G}^{-1}(j) \in[n]$ so that $G$ has a strand labeled $\ell \rightarrow j$. Then this strand $\ell \rightarrow j$ can be viewed as the intersection of $P_{j}$ with $H_{i}$. Let us explain this more rigorously. Let $\mathcal{R}, \mathcal{L} \subset\left(\begin{array}{c}{[n]} \\ k\end{array}\right)$ be the collections of sets in $\operatorname{Vert}\left(\Delta_{i}\right)$ lying on the right, resp., left side of the strand $\ell \rightarrow j$. So

$$
\mathcal{R}=\left\{S \in \operatorname{Vert}\left(\Delta_{i}\right) \mid j \notin S\right\} ; \quad \mathcal{L}=\{T \in \operatorname{Vert}(\Delta) \mid j \in T\} .
$$

From this one easily observes that $\mathcal{R}$, resp., $\mathcal{L}$ are the collections of sets in $\operatorname{Vert}\left(\Delta_{i}\right)$ that are on the negative, resp., positive sides of $P_{j}$. Finally, by our assumption on the normal of $P_{j}$ not being vertical, it follows that $P_{j} \cap \mathcal{Z}(n, 3) \cap H_{i}$ is a simple curve that divides the polygon $\mathcal{Z}(n, 3) \cap H_{i}$ into two parts in essentially the same way as the strand $\ell \rightarrow j$.

Finally we consider the well-studied notion of mutations of zonotopal tilings. It is easy to see that there are only two fine zonotopal tilings of $\mathcal{Z}(4,3)$. Given any two distinct fine zonotopal tilings $\Delta$ and $\Delta^{\prime}$ of $\mathcal{Z}(n, 3)$, we say that they differ by a mutation if there is a set $S \subset[n]$ 

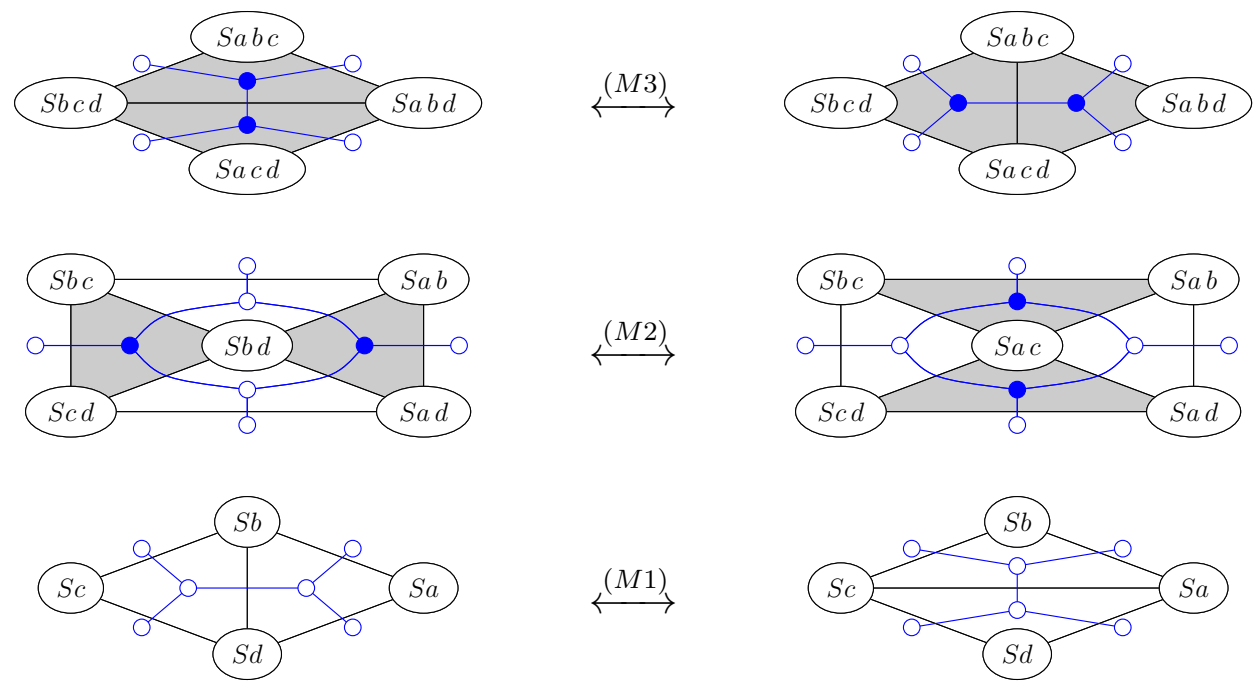

Figure 6. A mutation $\Delta \leftrightarrow \Delta^{\prime}$ corresponds to performing three simultaneous moves on plabic graphs: (M1) on level $|S|+1$, (M2) on level $|S|+2$, and (M3) on level $|S|+3$.

and four indices $a, b, c, d \in[n]-S$ such that for any tile $\tau_{X}$ we have

$$
X \in \Delta \Longleftrightarrow X \in \Delta^{\prime}
$$

unless $S \subset X^{+} \subset$ Sabcd and $X^{0} \subset\{a, b, c, d\}$. This can be reformulated as follows. Consider a signed set $Y=(S,[n]-S a b c d)$. Thus $\tau_{Y}$ is a zonotope that is combinatorially equivalent to $\mathcal{Z}(4,3)$. Hence there are only two fine zonotopal tilings of $\tau_{Y}$ which we denote $\Delta_{0}$ and $\Delta_{0}^{\prime}$ (the tilings $\Delta_{0}$ and $\Delta_{0}^{\prime}$ are shown in Figure 6). Then $\Delta$ and $\Delta^{\prime}$ differ by a mutation if and only if they coincide on the complement of $\tau_{Y}$ and their restrictions to $\tau_{Y}$ are $\Delta_{0}$ and $\Delta_{0}^{\prime}$ respectively.

As Figure 6] suggests, performing a mutation on $\Delta$ is equivalent to performing moves (M1), (M2), and (M3) on the corresponding plabic graphs.

Remark 3.1. For plabic graphs $G$ with $\pi_{G}=\sigma^{(k, n)}$, our construction allows to deduce Postnikov's Theorem 2.4 from Ziegler's 15, Theorem $4.1(\mathrm{G})$ ] where he shows that the higher Bruhat order $B(n, k)$ is a graded poset with a unique minimal and maximal elements. Indeed, fine zonotopal tilings of $\mathcal{Z}(n, 3)$ correspond to elements of $B(n, 2)$, and their mutations correspond to covering relations in $B(n, 2)$. 


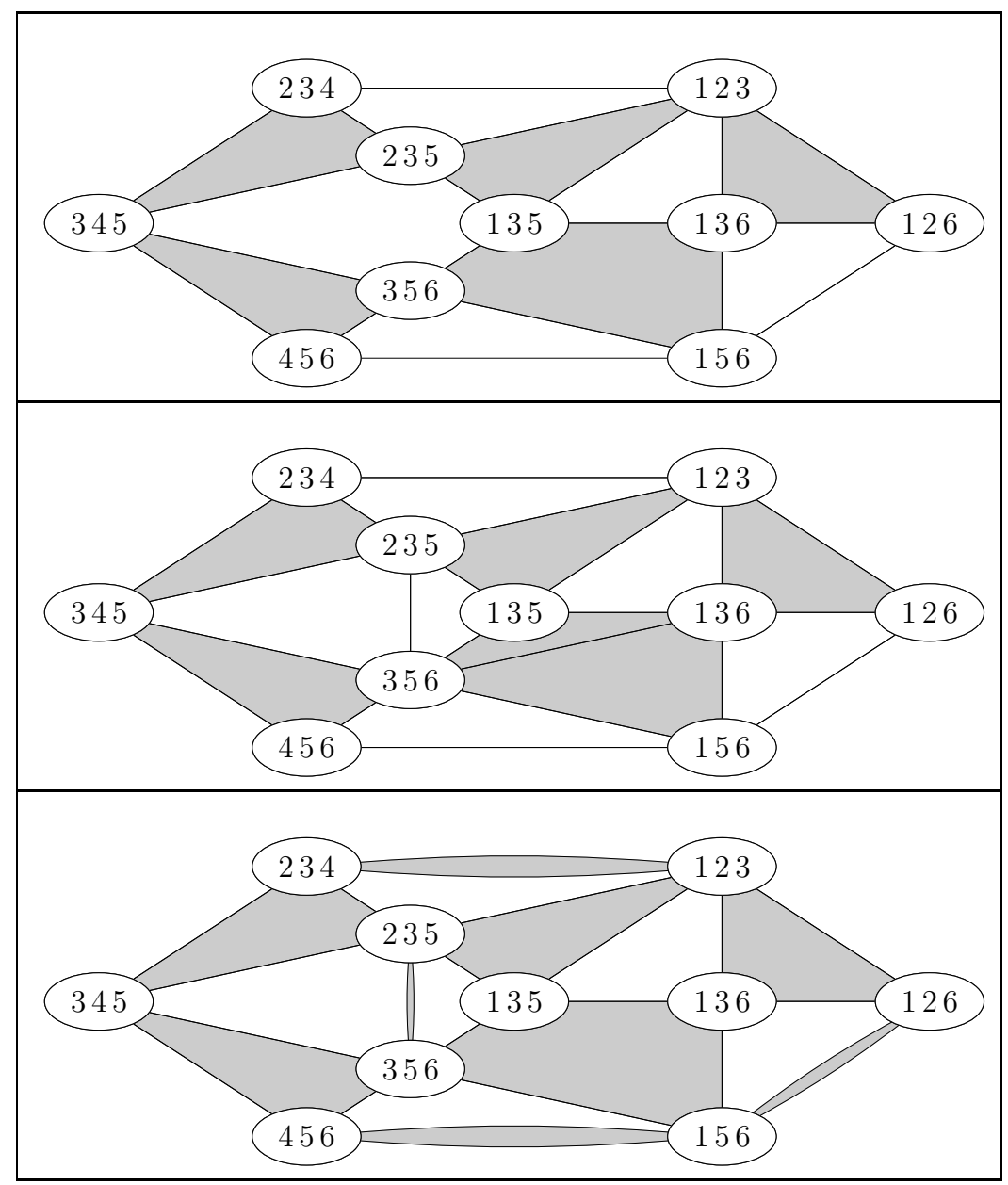

Figure 7. A maximal by inclusion chord separated collection in $\left(\begin{array}{c}{[6]} \\ 3\end{array}\right)$ corresponds to a plabic tiling (top). A triangulated plabic tiling (middle). A modified plabic tiling $\widetilde{\Sigma}_{i}^{\prime}$ from the proof of Lemma 4.2 (bottom).

\section{Proof of Theorem 1.2}

We now introduce a new object that is dual to reduced trivalent plabic graphs from Section 2.2.

Definition 4.1. A triangulated plabic tiling $\widetilde{\Sigma}$ corresponding to a maximal by inclusion chord separated collection $\mathcal{D} \subset\left(\begin{array}{c}{[n]} \\ k\end{array}\right)$ is any triangulation of $\Sigma(\mathcal{D})$ with the same set of vertices as $\Sigma(\mathcal{D})$. In other words, $\widetilde{\Sigma}$ consists of $\Sigma(\mathcal{D})$ together with a triangulation of each face of $\Sigma(\mathcal{D})$ corresponding to a non-trivial clique of size at least 4 . In this case, $k$ is called the level of $\widetilde{\Sigma}$. 
For example, Figure 7 (middle) contains one of the four possible triangulated plabic tilings corresponding to the collection in Figure 7 (top). Note that if an edge of a triangulated plabic tiling connects $\mathbf{v}_{S}$ and $\mathbf{v}_{T}$ then $S=T j-i$ and $T=S i-j$ for some $i \neq j$.

Each triangulated plabic tiling $\widetilde{\Sigma}$ has the underlying chord separated collection $\mathcal{D}$, and the vertices of $\widetilde{\Sigma}$ are naturally labeled by the elements of $\mathcal{D}$. We denote by $\operatorname{Vert}(\widetilde{\Sigma})$ the collection of such labels, thus $\mathcal{D}=$ $\operatorname{Vert}(\widetilde{\Sigma})$ can be reconstructed from $\widetilde{\Sigma}$ in a trivial manner.

Lemma 4.2. If $\widetilde{\Sigma}_{i}$ is a triangulated plabic tiling of level $i$ then the collection

$$
\mathrm{UP}\left(\widetilde{\Sigma}_{i}\right):=\left\{S \cup T \mid\left(\mathbf{v}_{S}, \mathbf{v}_{T}\right) \text { is an edge of } \widetilde{\Sigma}_{i}\right\}
$$

has size $(i+1)(n-i-1)+1$. Similarly, the collection

$$
\operatorname{DOWN}\left(\widetilde{\Sigma}_{i}\right):=\left\{S \cap T \mid\left(\mathbf{v}_{S}, \mathbf{v}_{T}\right) \text { is an edge of } \widetilde{\Sigma}_{i}\right\}
$$

has size $(i-1)(n-i+1)+1$.

Proof. The relation of chord separation is preserved under taking complements, so we only need to prove the first claim.

Take $\widetilde{\Sigma}_{i}$ and consider a pair of white triangles sharing an edge. Replace this edge by a pair of parallel edges and a black 2-gon inside. Do this for all such pairs, and also do the same thing for each boundary edge adjacent to a white triangle. For each pair of black triangles sharing an edge, remove that edge. Let $\widetilde{\Sigma}_{i}^{\prime}$ be the resulting modified tiling, which is a planar graph with faces colored black and white. Each of its non-boundary edges lies between a white face and a black face. Such a modified tiling $\widetilde{\Sigma}_{i}^{\prime}$ is illustrated in Figure 7 (bottom).

The sets in $\operatorname{UP}\left(\widetilde{\Sigma}_{i}\right)$ correspond naturally to the black faces of $\widetilde{\Sigma}_{i}^{\prime}$. All the white faces of $\widetilde{\Sigma}_{i}^{\prime}$ are triangles, and thus the number of edges in $\widetilde{\Sigma}_{i}^{\prime}$ equals $3 w+n$, where $w$ is the number of white faces in $\widetilde{\Sigma}_{i}^{\prime}$ and $n$ is the number of boundary edges in $\widetilde{\Sigma}_{i}^{\prime}$ (by construction, all of them are adjacent to a black face). Let $b$ be the number of black faces of $\widetilde{\Sigma}_{i}^{\prime}$, so $\left|\operatorname{UP}\left(\widetilde{\Sigma}_{i}\right)\right|=b$ is the number that we want to find. By Theorem 2.7, we know that the number of vertices of $\widetilde{\Sigma}_{i}^{\prime}$ equals $i(n-i)+1$.

It follows from the general theory of plabic graphs (see [11]) that the number of white triangles in $\widetilde{\Sigma}_{i}^{\prime}$ equals $w=i(n-i-1)$. Indeed, $w$ is the number of trivalent white vertices in a reduced plabic graph corresponding to the top cell, and this number is invariant under square moves and contractions/uncontractions, so one just needs to count this number for the corresponding I diagram, which in this case is a rectangle, see [11, Figure 20.1]. 
We can now apply Euler's formula, where we also need to count the outer face:

$$
i(n-i)+1-(3 w+n)+(w+b+1)=2 .
$$

This is an equation on $b$ yielding the desired result $b=(i+1)(n-i-$ $1)+1$. As an example, take the tiling from the bottom of Figure 7. We have $i=3, n=6, w=6, b=9$ so the above equation is indeed satisfied:

$$
3 \cdot 3+1-(3 \cdot 6+6)+(6+9+1)=10-24+16=2 .
$$

Definition 4.3. Let $\widetilde{\Sigma}_{i}, \widetilde{\Sigma}_{i+1}$ be any two triangulated plabic tilings of levels $i$ and $i+1$ respectively. We say that the triangulated plabic tilings $\widetilde{\Sigma}_{i}, \widetilde{\Sigma}_{i+1}$ are compatible if

- for every edge $\left(\mathbf{v}_{S}, \mathbf{v}_{T}\right)$ of $\widetilde{\Sigma}_{i}, \mathbf{v}_{S \cup T}$ is a vertex of $\widetilde{\Sigma}_{i+1}$;

- for every edge $\left(\mathbf{v}_{S}, \mathbf{v}_{T}\right)$ of $\widetilde{\Sigma}_{i+1}, \mathbf{v}_{S \cap T}$ is a vertex of $\widetilde{\Sigma}_{i}$.

Lemma 4.2 combined with Theorem 2.7 yields the following:

Corollary 4.4. Let $\widetilde{\Sigma}_{i}, \widetilde{\Sigma}_{i+1}$ be two compatible triangulated plabic tilings of levels $i$ and $i+1$ respectively. Then

$$
\operatorname{Vert}\left(\widetilde{\Sigma}_{i+1}\right)=\operatorname{UP}\left(\widetilde{\Sigma}_{i}\right) ; \quad \operatorname{Vert}\left(\widetilde{\Sigma}_{i}\right)=\operatorname{DOWN}\left(\widetilde{\Sigma}_{i+1}\right) .
$$

Proof. By definition, the collection $\operatorname{Vert}\left(\widetilde{\Sigma}_{i+1}\right)$ contains $\operatorname{UP}\left(\widetilde{\Sigma}_{i}\right)$. By Lemma 4.2, their sizes coincide.

By definition, the collection $\operatorname{Vert}\left(\widetilde{\Sigma}_{i}\right)$ is chord separated (and is maximal by inclusion in $\left(\begin{array}{c}{[n]} \\ i\end{array}\right)$ ). It turns out that given two compatible tilings, their vertices are chord separated from each other as well:

Lemma 4.5. Let $\widetilde{\Sigma}_{i}, \widetilde{\Sigma}_{i+1}$ be two compatible triangulated plabic tilings of levels $i$ and $i+1$ respectively. Then the collection $\operatorname{Vert}\left(\widetilde{\Sigma}_{i}\right) \cup \operatorname{Vert}\left(\widetilde{\Sigma}_{i+1}\right)$ is chord separated.

Proof. Suppose that $T \in \operatorname{Vert}\left(\widetilde{\Sigma}_{i}\right)$ and $S \in \operatorname{Vert}\left(\widetilde{\Sigma}_{i+1}\right)$ are not chord separated, that is, there exist cyclically ordered integers $a, b, c, d \in[n]$ such that $a, c \in S-T, b, d \in T-S$. By Corollary 4.4, there is an edge in $\widetilde{\Sigma}_{i+1}$ connecting $T x$ and $T y$ for some $x \neq y \notin T$. Both of these sets have to be chord separated from $S$ and the only way for this to be possible is if $x=a$ and $y=c$ (or vice versa). Note that $|T x y|=i+2$ and $|S|=i+1$, but $b, d \in T x y-S$. It means that $S \not \subset T x y$, so let $z \in S-T x y$. And because $x=a$ and $y=c$, we have $z \neq a, b, c, d$. If $z$ belongs to the cyclic interval $(d, b)$ then $S$ is not chord separated from 
$T x=T a$. Otherwise $z$ belongs to the cyclic interval $(b, d)$ in which case $S$ is not chord separated from $T y=T c$. This contradiction finishes the proof of the lemma.

A family $\widetilde{\Sigma}_{*}:=\left(\widetilde{\Sigma}_{0}, \widetilde{\Sigma}_{1}, \ldots, \widetilde{\Sigma}_{n}\right)$ of triangulated plabic tilings such that the level of $\widetilde{\Sigma}_{i}$ is equal to $i$ and $\widetilde{\Sigma}_{i}$ and $\widetilde{\Sigma}_{i+1}$ are compatible for all $i=0, \ldots, n-1$ is called admissible.

Now we go back to collections $\mathcal{D} \subset 2^{[n]}$ without any restrictions on sizes. Given such a collection, for all $i=0,1, \ldots, n$ denote $\mathcal{D}^{(i)}:=\mathcal{D} \cap$

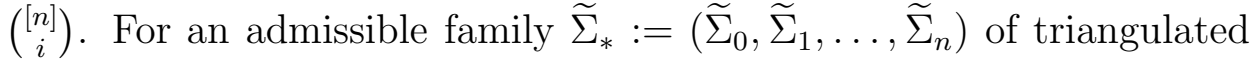
plabic tilings, denote

$$
\operatorname{Vert}\left(\widetilde{\Sigma}_{*}\right)=\cup_{i=0}^{n} \operatorname{Vert}\left(\widetilde{\Sigma}_{i}\right)
$$

to be the set of all vertex labels of tilings in $\widetilde{\Sigma}_{*}$.

Proposition 4.6. The map $\widetilde{\Sigma}_{*} \mapsto \operatorname{Vert}\left(\widetilde{\Sigma}_{*}\right)$ is a bijection between admissible families of triangulated plabic tilings and maximal by inclusion chord separated collections in $2^{[n]}$. In particular, if $\mathcal{D} \subset 2^{[n]}$ is any maximal by inclusion chord-separated collection then $\mathcal{D}^{(i)} \subset\left(\begin{array}{c}{[n]} \\ i\end{array}\right)$ is also maximal by inclusion (and by size), and if $\mathcal{D}=\operatorname{Vert}\left(\widetilde{\Sigma}_{*}\right)$ then

$$
\mathcal{D}^{(i)}=\operatorname{Vert}\left(\widetilde{\Sigma}_{i}\right)
$$

Before we proceed to the proof, let us explain the connection between admissible families of triangulated plabic tilings and zonotopal tilings.

Consider the zonotope $\mathcal{Z}(n, 3)$ and its fine zonotopal tiling $\Delta$. Recall that for each $i=0, \ldots, n, H_{i}$ is the plane given by $z=i$. Denote $\mathcal{Z}(n, 3)^{(i)}:=\mathcal{Z}(n, 3) \cap H_{i}$ and $\Delta^{(i)}:=\left\{\tau_{X} \cap H_{i} \mid X \in \Delta\right\}$. It is clear that $\mathcal{Z}(n, 3)^{(i)}$ is a convex polygon and $\Delta^{(i)}$ is a triangulation of $\mathcal{Z}(n, 3)^{(i)}$ (which may have some vertices inside $\left.\mathcal{Z}(n, 3)^{(i)}\right)$. Moreover, by definition, each vertex of $\Delta$, and thus of $\Delta^{(i)}$, is labeled by a subset of $[n]$.

Proposition 4.7. The map $\Delta \mapsto\left(\Delta^{(i)}\right)_{i=0}^{n}$ is a bijection between fine zonotopal tilings of $\mathcal{Z}(n, 3)$ and admissible families of triangulated plabic tilings.

Proof. First we show that $\left(\Delta^{(i)}\right)_{i=0}^{n}$ is an admissible family of triangulated plabic tilings. Let $\mathcal{D}:=\operatorname{Vert}(\Delta)$ and suppose that $\mathcal{D}$ is not a chord separated collection. Then there must exist integers $a<b<c<$ $d \in[n]$ and two sets $S, T \in \mathcal{D}$ such that $a, c \in S-T$ and $b, d \in T-S$. Recall from Section 3 that fine zonotopal tilings of $\mathcal{Z}(n, 3)$ are dual to arrangements of pseudoplanes. Let $\left(P_{1}, \ldots, P_{n}\right)$ be the pseudoplane arrangement dual to $\Delta$. Since $(\{a, c\},\{b, d\})$ is a Radon partition of 
of $\mathbf{C}(n, 3)$, the pseudoplanes $P_{a}, P_{b}, P_{c}, P_{d}$ bound a certain simplicial region $R$ in $\mathbb{R}^{3}$, and both vertices $\mathbf{v}_{S}$ and $\mathbf{v}_{T}$ have to be inside $R$. This leads to a contradiction because if $S$ and $T$ belong to the same region, their restrictions on $\{a, b, c, d\}$ must also coincide. A more rigorous argument can be given using Ziegler's bijection 1 between fine zonotopal tilings of $\mathcal{Z}(n, 3)$ and consistent subsets of $\left(\begin{array}{c}{[n]} \\ 4\end{array}\right)$. One easily observes that under this bijection, $\phi(\Delta)$ contains $\{a, b, c, d\}$ if and only if there is a subset $U \in \operatorname{Vert}(\Delta)$ with $a, c \in U$ and $b, d \notin U$. Similarly, $\phi(\Delta)$ does not contain $\{a, b, c, d\}$ if and only if there is a subset $U \in \operatorname{Vert}(\Delta)$ with $b, d \in U$ and $a, c \notin U$. The fact that $\phi(\Delta)$ is well defined implies that $S$ and $T$ cannot both appear in $\operatorname{Vert}(\Delta)$.

A simple deletion-contraction argument shows that the number of vertices of $\Delta$ equals the number of independent sets of the corresponding oriented matroid. In other words, the vertex labels $\mathcal{D}:=\operatorname{Vert}(\Delta)$ form a chord-separated collection in $2^{[n]}$ satisfying

$$
|\mathcal{D}|=\left(\begin{array}{l}
n \\
0
\end{array}\right)+\left(\begin{array}{l}
n \\
1
\end{array}\right)+\left(\begin{array}{l}
n \\
2
\end{array}\right)+\left(\begin{array}{l}
n \\
3
\end{array}\right) .
$$

Note that since $\mathcal{D}$ is a chord separated collection, $\mathcal{D}^{(i)}$ is a weakly separated collection in $\left(\begin{array}{c}{[n]} \\ i\end{array}\right)$, and hence has size at most $i(n-i)+1$. Summing over all $i$, we get

$$
\sum_{i=0}^{n}\left|\mathcal{D}^{(i)}\right| \leq \sum_{i=0}^{n}(i(n-i)+1)=\frac{n^{3}+5 n+1}{6} .
$$

One easily checks that this is equal to

$$
\left(\begin{array}{l}
n \\
0
\end{array}\right)+\left(\begin{array}{l}
n \\
1
\end{array}\right)+\left(\begin{array}{l}
n \\
2
\end{array}\right)+\left(\begin{array}{l}
n \\
3
\end{array}\right)=|\mathcal{D}| .
$$

Thus all the inequalities become equalities and each $\mathcal{D}^{(i)}$ is a maximal by size weakly separated collection in $\left(\begin{array}{c}{[n]} \\ i\end{array}\right)$.

Next, observe that for each top-dimensional zonotope $\tau_{X}$ in $\Delta$, the set $X^{0}$ has exactly three elements, say, $a, b, c \in[n]$, and thus all vertices of $\tau_{X}$ have the form

$$
\operatorname{Vert}\left(\tau_{X}\right)=\left\{\mathbf{v}_{S}, \mathbf{v}_{S a}, \mathbf{v}_{S b}, \mathbf{v}_{S c}, \mathbf{v}_{S a b}, \mathbf{v}_{S b c}, \mathbf{v}_{S a c}, \mathbf{v}_{S a b c}\right\}
$$

for $S=X^{+}$. Denote $k:=|S|$, then the only non-empty intersections of $\tau_{X}$ with planes $H_{i}$ happen for $i=k, k+1, k+2, k+3$. Moreover, for $i=k$ and $i=k+3$, the intersection is just a single vertex, and for $i=k+1, k+2$, the intersection is a triangle. For example, for

\footnotetext{
${ }^{1}$ this is basically an application of Bohne-Dress theorem: $\Delta$ defines a localization in general position of the dual matroid, and this localization is the same thing as $\phi(\Delta)$ viewed as a map from the cocircuits of the dual matroid to $\{+,-\}$.
} 
$i=k+1$, this triangle has vertices $\mathbf{v}_{S a}, \mathbf{v}_{S b}, \mathbf{v}_{S c}$, and thus is a subset of the corresponding white clique $\mathcal{W}(S)$ in $\Sigma\left(\mathcal{D}^{(i)}\right)$. Similarly, for $i=k+2$, the triangle lies inside a black clique $\mathcal{B}(S a b c)$ in $\Sigma\left(\mathcal{D}^{(i)}\right)$. Thus $\left(\Delta^{(i)}\right)_{i=0}^{n}$ indeed form a family of triangulated plabic tilings. For every edge connecting, for example, $\mathbf{v}_{S a}$ and $\mathbf{v}_{S b}, \mathbf{v}_{S a b}$ is a vertex on level $k+2$ and $\mathbf{v}_{S}$ is a vertex on level $k$, and similarly, for every edge of the form $\left(\mathbf{v}_{S a b}, \mathbf{v}_{S b c}\right), \mathbf{v}_{S b}$ is a vertex on level $k+1$ and $\mathbf{v}_{S a b c}$ is a vertex on level $k+3$. Thus the family $\left(\Delta^{(i)}\right)_{i=0}^{n}$ is admissible, and we have finished one direction of the proposition.

Now assume that we are given an admissible family $\widetilde{\Sigma}_{*}$ of triangulated plabic tilings, and we want to construct a fine zonotopal tiling $\Delta$ of $\mathcal{Z}(n, 3)$ satisfying $\Delta^{(i)}=\widetilde{\Sigma}_{*}^{(i)}$ for all $i=0,1, \ldots, n$. It suffices to describe the top-dimensional tiles $\tau_{X}$ of $\Delta$. As before, each such tile can be indexed by a subset $S=X^{+}$and three elements $a, b, c$ with $\{a, b, c\}=X^{0}$, so we denote it $\tau_{S, a, b, c}$. We are ready to describe all top-dimensional faces of $\Delta$ : they are all faces of the form $\tau_{S, a, b, c}$ where $S a, S b$, and $S c$ are the vertex labels of a white triangle in one of the tilings in $\widetilde{\Sigma}_{*}$.

So far, we only know that thus defined $\Delta$ is a collection of parallelotopes, and we need to see why their union is the whole $\mathcal{Z}(n, 3)$ and why the intersection of any two of them is their common face. We start with the latter.

Claim: The intersection of any two tiles is in $\Delta$ is either empty or their common face.

Proof: We will only consider the case of two top-dimensional tiles intersecting in their relative interior, the case of lower-dimensional tiles is handled similarly. Consider two tiles $\tau_{S, a, b, c}$ and $\tau_{T, d, e, f}$ and suppose that their interiors intersect on some point $p \in \tau_{S, a, b, c} \cap \tau_{T, d, e, f}$. Then there exist numbers $0<\lambda_{a}, \lambda_{b}, \lambda_{c}, \mu_{d}, \mu_{e}, \mu_{f}<1$ satisfying

(1) $\sum_{s \in S} 1 \cdot \mathbf{v}_{s}+\lambda_{a} \mathbf{v}_{a}+\lambda_{b} \mathbf{v}_{b}+\lambda_{c} \mathbf{v}_{c}=\sum_{t \in T} 1 \cdot \mathbf{v}_{t}+\mu_{d} \mathbf{v}_{d}+\mu_{e} \mathbf{v}_{e}+\mu_{f} \mathbf{v}_{f}$.

We are going to construct two subsets $S^{\prime} \in \operatorname{Vert}\left(\tau_{S, a, b, c}\right)$ and $T^{\prime} \in$ $\operatorname{Vert}\left(\tau_{T, d, e, f}\right)$ that are not chord separated but such that their sizes differ by at most 1 . Let $X=\left(X^{+}, X^{-}\right)$be the signed vector defined as follows: $X^{+}$contains all elements $g$ of $[n]$ such that the coefficient of $\mathbf{v}_{g}$ in the left hand side of (11) is strictly greater than the coefficient of $\mathbf{v}_{g}$ in the right hand side of (11). The set $X^{-}$is defined similarly, replacing left with right. Since the left hand side equals the right hand side, there exist cyclically ordered integers $p, q, r, s$ such that $C^{+}:=\{p, r\} \subset X^{+}$ 
and $C^{-}:=\{q, s\} \subset X^{-}$(such a signed set $C:=\left(C^{+}, C^{-}\right)$is called a Radon partition of $\mathbf{C}(n, 3))$.

It is clear that $C^{+} \subset S a b c-T$ while $C^{-} \subset T$ def $-S$. Thus there exists at least one pair of subsets $S^{\prime} \in \tau_{S, a, b, c}$ and $T^{\prime} \in \tau_{T, d, e, f}$ such that $C^{+} \subset S^{\prime}-T^{\prime}$ and $C^{-} \subset T^{\prime}-S^{\prime}$. We want to choose $S^{\prime}$ and $T^{\prime}$ so that their levels differ by at most 1 . Suppose this cannot be done, and that the level of any such $S^{\prime}$ is, say, greater than the level of any such $T^{\prime}$ by at least 2. Then we set $S^{\prime}:=S \cup C^{+}$and $T^{\prime}:=T$ def $-C^{+}$. These two sets clearly satisfy $C^{+} \subset S^{\prime}-T^{\prime}$ and $C^{-} \subset T^{\prime}-S^{\prime}$, so if we show that $\left|S^{\prime}\right| \leq\left|T^{\prime}\right|+1$ then we will get a contradiction.

Let $h$ be the sum of the coefficients in any of the sides of (11) (they are equal since every vector from $\mathbf{C}(n, 3)$ has height 1$)$. If $h$ is an integer then the bad intersection would happen inside one triangulated plabic tiling which is impossible by Theorem 2.7. Thus let $h^{-}<h<h^{+}$be the floor and the ceiling of $h$.

We are going to consider a number of cases:

(1) $\left|S^{\prime}\right| \leq h^{+}$and $\left|T^{\prime}\right| \geq h^{-}$

(2) $\left|T^{\prime}\right|<h^{-}$

(3) $\left|S^{\prime}\right|>h^{+}$.

For each of them, we need to show that $\left|S^{\prime}\right| \leq\left|T^{\prime}\right|+1$. Case (1) implies that trivially. Cases (2) and (3) are symmetric with respect to taking complements of all sets, so we only need to consider one of them. From now on, we assume that $\left|S^{\prime}\right|>h^{+}$. Recall that $S^{\prime}=S \cup C^{+}$where $C^{+}$is a two-element set. Thus $\left|S^{\prime}\right| \leq|S|+2$. Now, the integers $|S|, h^{+}$, and $\left|S^{\prime}\right|$ satisfy (since $|S| \leq h$ and $h<h^{+}$)

$$
|S|<h^{+}<\left|S^{\prime}\right| \leq|S|+2
$$

which implies that

$$
|S|=h^{-} ; \quad|S|+1=h^{+} ; \quad|S|+2=\left|S^{\prime}\right| .
$$

In particular, the last equality implies that $C^{+} \cap S=\emptyset$, in other words, that $C^{+} \subset\{a, b, c\}$. Without loss of generality we may assume that $C^{+}=\{a, b\}$. It follows that $\lambda_{a}+\lambda_{b}<h-|S|$ because

$$
h=|S|+\lambda_{a}+\lambda_{b}+\lambda_{c}>|S|+\lambda_{a}+\lambda_{b} .
$$

Our goal is to show that $\left|T^{\prime}\right| \geq h^{+}$. We are going to consider three cases:

(a) $\left|C^{+} \cap\{d, e, f\}\right|=2$;

(b) $\left|C^{+} \cap\{d, e, f\}\right|=1$;

(c) $\left|C^{+} \cap\{d, e, f\}\right|=0$;

First consider case (国), say, $d=a$ and $e=b$. By definition, we then have $\left|T^{\prime}\right|=|T|+1$. Since $a, b \in C^{+} \subset X^{+}$, the coefficients of $\mathbf{v}_{a}$ and 
$\mathbf{v}_{b}$ in the right hand side are less than the corresponding coefficients in the left hand side. We get that

$$
\mu_{d}+\mu_{e}<\lambda_{a}+\lambda_{b}<h-|S| \text {. }
$$

But this implies that $|T| \geq|S|$, because otherwise if $|T| \leq|S|-1$ then

$$
h=|T|+\mu_{d}+\mu_{e}+\mu_{f}<|S|-1+(h-|S|)+\mu_{f}<h,
$$

which is impossible. Thus $|T| \geq|S|=h^{-}$and since $\left|T^{\prime}\right|=|T|+1$, we get that $\left|T^{\prime}\right| \geq h^{+}$. We are done with case (a) .

Now consider case (b) , say, $d=a$. Then by definition, $\left|T^{\prime}\right|=|T|+2$. Also, since $a \in C^{+} \subset X^{+}$, we have $\mu_{d}<\lambda_{a}<h-|S|$. We need to show $\left|T^{\prime}\right| \geq h^{+}$, equivalently, $|T| \geq|S|-1$. This is indeed true, because otherwise if $|T| \leq|S|-2$ then

$$
h=|T|+\mu_{d}+\mu_{e}+\mu_{f}<|S|-2+(h-|S|)+\mu_{e}+\mu_{f}<h,
$$

which is impossible. Thus we are done with the case (b), and the case (ㄷ) is trivial since for this case, $\left|T^{\prime}\right|=|T|+3>h$ and so $\left|T^{\prime}\right| \geq h^{+}$. We have finished the proof of the fact that $S^{\prime}$ and $T^{\prime}$ can be chosen so that their levels differ by at most 1 . But they are not chord separated which contradicts Lemma 4.5 .

Claim: The union $U$ of all tiles in $\Delta$ equals $\mathcal{Z}(n, 3)$.

Proof: Observe that $U$ is a closed polyhedral complex inside $\mathcal{Z}(n, 3)$ that contains the boundary of $\mathcal{Z}(n, 3)$, because the vertices of the boundary are labeled by all cyclic intervals.

Assume that $U \subsetneq \mathcal{Z}(n, 3)$ and let $p \in \mathcal{Z}(n, 3)-U$ be any point. Choose a generic point $q$ in the interior of any tile of $\Delta$, and draw a segment $[p, q]$. Let $\tau_{X}$ be the closest to $p$ 2-dimensional face of $\Delta$ that intersects $[p, q]$, and let $r$ be their intersection point. Such a face has vertices $\mathbf{v}_{S}, \mathbf{v}_{S a}, \mathbf{v}_{S b}, \mathbf{v}_{S a b}$ for some $S \subset[n]$ and $a, b \in[n]$. But then there is an edge between $\mathbf{v}_{S a}$ and $\mathbf{v}_{S b}$ in the corresponding triangulated plabic tiling $\widetilde{\Sigma}_{k}$ where $k=|S|+1$. This edge is the common edge of two triangles, and each of them belongs to a three-dimensional tile, so we can see that $\tau_{X}$ is the common face of these two tiles. Thus $r$ is contained in $U$ together with some open neighborhood, which leads to a contradiction, thus finishing the proof or the claim.

Proposition 4.7 follows from the two claims above.

Proof of Proposition 4.6. As we have shown in the proof of Proposition 4.7, if $\widetilde{\Sigma}_{*}$ is an admissible family of triangulated plabic tilings then $\operatorname{Vert}\left(\widetilde{\Sigma}_{*}\right)$ is a maximal by size chord separated collection in $2^{[n]}$. In particular, it is also maximal by inclusion, which shows one direction of the proposition. Assume now that we are given a maximal by inclusion chord separated collection $\mathcal{D} \subset 2^{[n]}$, and we need to reconstruct 
an admissible family $\widetilde{\Sigma}_{*}$ with $\operatorname{Vert}\left(\widetilde{\Sigma}_{*}\right)=\mathcal{D}$. Note that it is not even a priori clear why $\mathcal{D}^{(i)}$ is maximal by inclusion in $\left(\begin{array}{c}{[n]} \\ i\end{array}\right)$. But we claim that there exists a unique admissible family $\widetilde{\Sigma}_{*}$ such that:

- the vertex labels of $\widetilde{\Sigma}_{*}$ are precisely the sets in $\mathcal{D}$;

- $\left(\mathbf{v}_{S}, \mathbf{v}_{T}\right)$ is an edge of $\widetilde{\Sigma}_{i}$ if and only if

$$
|S|=|T|=i,|S \cap T|=i-1,|S \cup T|=i+1 \text {, and } S \cap T, S \cup T \in \mathcal{D} \text {. }
$$

We are going to reconstruct the triangulated plabic tilings $\widetilde{\Sigma}_{i}$ one by one for $i=0,1, \ldots, n$. For $i=0, \widetilde{\Sigma}_{0}$ consists of the unique vertex labeled by $\emptyset$. For $i=1$, all the one-element sets have to belong to $\mathcal{D}$ because it is maximal by inclusion and one-element sets are chord separated from all other sets. Thus $\mathcal{D}^{(1)}$ is a maximal by inclusion collection in $\left(\begin{array}{c}{[n]} \\ 1\end{array}\right)$. Our induction on $i$ is going to be based on the following lemma:

Lemma 4.8. Suppose that $\mathcal{D} \subset 2^{[n]}$ is a maximal by inclusion chord separated collection, and that there exists an $i \in[n]$ such that $\mathcal{D}^{(i)}$ is a maximal by inclusion collection in $\left(\begin{array}{c}{[n]} \\ i\end{array}\right)$, so that it corresponds to a plabic tiling $\Sigma\left(\mathcal{D}^{(i)}\right)$ via Theorem 2.7. Then (2) gives a triangulation of the polygons of $\Sigma\left(\mathcal{D}^{(i)}\right)$, therefore transforming it into a triangulated plabic tiling $\widetilde{\Sigma}_{i}$.

Let us first show that Lemmas 4.8 and 4.2 together imply Proposition 4.6. As we have already shown, $\mathcal{D}^{(1)}$ is a maximal by inclusion chord separated collection in $\left(\begin{array}{c}{[n]} \\ 1\end{array}\right)$. Then Lemma 4.8 gives a unique triangulated plabic tiling $\widetilde{\Sigma}_{1}$. But then Lemma 4.2 states that $\mathcal{D}^{(2)} \supset \operatorname{UP}\left(\widetilde{\Sigma}_{1}\right)$ is a maximal by size (and thus by inclusion) chord separated collection in $\left(\begin{array}{c}{[n]} \\ 2\end{array}\right)$, after which Lemma 4.8 produces $\widetilde{\Sigma}_{2}$. Continuing in this fashion, we reconstruct the whole family $\widetilde{\Sigma}_{*}$, and it is compatible by construction, while, clearly, $\operatorname{Vert}\left(\widetilde{\Sigma}_{*}\right)=\mathcal{D}$, which finishes the proof of Proposition 4.6.

Proof of Lemma 4.8. Let $\mathcal{D} \subset 2^{[n]}$ and $\mathcal{D}^{(i)} \subset\left(\begin{array}{c}{[n]} \\ i\end{array}\right)$ be maximal by inclusion chord separated collections. We want to show that (2) gives a triangulation of $\Sigma\left(\mathcal{D}^{(i)}\right)$. We are only going to show that it gives a triangulation of white cliques of $\Sigma\left(\mathcal{D}^{(i)}\right)$, for black cliques, one can just replace all sets in $\mathcal{D}$ by their complements and then apply the statement for white cliques.

Our first goal is to show that every edge $\left(\mathbf{v}_{S}, \mathbf{v}_{T}\right)$ of $\Sigma\left(\mathcal{D}^{(i)}\right)$ satisfies (2). 
Claim: Suppose $\mathcal{D} \subset 2^{[n]}$ and $\mathcal{D}^{(i)}$ are maximal by inclusion chord separated collections, and assume that $\mathcal{W}(S)$ is a non-trivial white clique in $\Sigma\left(\mathcal{D}^{(i)}\right)$. Then $S \in \mathcal{D}$.

Proof: Let $\mathcal{W}(S)=\left\{S a_{1}, S a_{2}, \ldots, S a_{r}\right\}$ for some $r \geq 3$. Suppose we have found $T \in \mathcal{D}$ and $a, b, c, d$ cyclically ordered such that $a, c \in T-S$ and $b, d \in S-T$. Since $r \geq 3$, we can find an index $j \in[r]$ such that $a_{j} \neq a, c$. Then $a, c \in T-S a_{j}$ and $b, d \in S a_{j}-T$ so $S a_{j}$ and $T$ are not chord separated which is impossible since they both belong to $\mathcal{D}$.

Claim: Suppose $\mathcal{D} \subset 2^{[n]}$ and $\mathcal{D}^{(i)}$ are maximal by inclusion chord separated collections, and assume that $\mathcal{B}(S)$ is a non-trivial black clique in $\Sigma\left(\mathcal{D}^{(i)}\right)$. Then $S \in \mathcal{D}$.

Proof: Follows from the previous claim by replacing all subsets with their complements.

From these two claims, it becomes clear why all edges of $\Sigma$ satisfy (2): each of them is either a boundary edge, in which case it connects two cyclic intervals so the result follows trivially, or it is an edge that separates a white clique from a black clique, and thus the intersection and the union of the vertex labels belong to $\mathcal{D}$ by the above claims.

So all edges of $\Sigma\left(\mathcal{D}^{(i)}\right)$ satisfy (2). Add all the other edges given by (2) to it to get a new tiling which we denote $\widetilde{\Sigma}^{\prime}$. It is clear that these new edges subdivide the polygons of $\Sigma\left(\mathcal{D}^{(i)}\right)$ into smaller polygons and do not intersect each other or the edges of $\Sigma\left(\mathcal{D}^{(i)}\right)$.

Let $A:=\left\{a_{1}<a_{2}<\cdots<a_{r}\right\} \subset[n]$ and put $a_{r+1}:=a_{1}$. Suppose $S$ is an $i$-1-element subset of $[n]-A$ such that:

- $\left\{S a_{1}, \ldots, S a_{r}\right\} \subset \mathcal{W}(S) \subset \mathcal{D}^{(i)}$;

- $\left\{S a_{1}, \ldots, S a_{r}\right\}$ are the vertex labels of a (white) polygon $P$ of $\widetilde{\Sigma^{\prime}}$;

- $\left(S a_{i}, S a_{i+1}\right)$ is an edge of $P$ for every $i=1,2, \ldots, r$;

- $P$ has no other edges inside.

We assume that $r>3$ and we would like to see why the polygon $P$ actually has to have an edge inside.

We are going to start with removing all the irrelevant elements. Define a new collection $\mathcal{D}^{\prime} \subset 2^{[r]}$ as follows:

$$
\mathcal{D}^{\prime}:=\left\{T^{\prime} \subset[r] \mid S \cup\left\{a_{j}\right\}_{j \in T^{\prime}} \in \mathcal{D}\right\} .
$$

Claim: The collection $\mathcal{D}^{\prime} \subset 2^{[r]}$ is a maximal by inclusion chord separated collection.

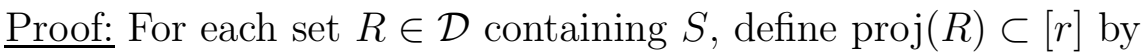

$$
\operatorname{proj}(R):=\left\{j \in[r] \mid a_{j} \in R\right\}
$$


Now we define another collection of sets

$$
\mathcal{D}^{\prime \prime}:=\{\operatorname{proj}(R) \mid S \subset R \text { and } R \in \mathcal{D}\} .
$$

It is clear that $\mathcal{D}^{\prime}$ and $\mathcal{D}^{\prime \prime}$ are chord separated and $\mathcal{D}^{\prime} \subset \mathcal{D}^{\prime \prime}$. We are going to show that if a set $T^{\prime} \subset[r]$ is chord separated from all $\mathcal{D}^{\prime \prime}$ then $T^{\prime} \in \mathcal{D}^{\prime}$. If we manage to do this, it will follow that $\mathcal{D}^{\prime}=\mathcal{D}^{\prime \prime}$ are both maximal by inclusion chord separated collections.

Suppose that there exists a set $T^{\prime} \subset[r]$ which is chord separated from all elements of $\mathcal{D}^{\prime \prime}$ but $T:=S \cup\left\{a_{j}\right\}_{j \in T^{\prime}}$ is not chord separated from at least one set $R \in \mathcal{D}$. Let $a, b, c, d$ be cyclically ordered elements of $[n]$ such that $a, c \in R-T$ and $b, d \in T-R$. Our ultimate goal is to show that $S \subset R$ and that $\operatorname{proj}(R)$ and $T^{\prime}$ are not chord separated.

Since $T \supset S$, we have $a, c, \notin S$. Next, $b, d \in A$ : otherwise, if at least one of them, say, $b$, is not in $A$ (and therefore is in $S$ because $S \subset T \subset S \cup A$ ) then $b, d \in S d-R$ and $a, c \in R-S d$ which contradicts the fact that both of them belong to $\mathcal{D}$. Thus $b, d \in A$, so $S b, S d \in \mathcal{D}$. Now assume that there is an element $s \in S-R$. Then $s \neq a, c$ and so $s$ belongs to one of the circuilar intervals: either $s \in(a, c)$ or $s \in(c, a)$. We know that $b \in(a, c)$ and $d \in(c, a)$ and thus if $s \in(a, c)$ then $S d$ is not chord separated from $R$, and if $s \in(c, a)$ then $S b$ is not chord separated from $R$, and in any case we get a contradiction, which means $S \subset R$ and we are half way there.

If $a, c \in A$ then we are done, $\operatorname{because} \operatorname{proj}(R)$ and $T^{\prime}$ would obviously not be chord separated in this case. Assume that $a \notin A$ and let $j \in[r]$ be such that $a$ belongs to the cyclic interval $\left(a_{j}, a_{j+1}\right)$. Since $b, d \in A$ are strictly between $a$ and $c$, we have that $c \in\left(a_{j+1}, a_{j}\right)$. The set $S a_{j} a_{j+1}$ belongs to $\mathcal{D}$, and $c \in R-S a_{j} a_{j+1}$, which means that either $a_{j}$ or $a_{j+1}$ belongs to $R$. Suppose this is $a_{j}$, and then $a_{j} \neq b, d$ so we can just replace $a$ with $a_{j}$ while still preserving the fact that $a, b, c, d$ are cyclically ordered and $a, c \in R-T$ while $b, d \in T-R$. After we do the same with $c$, we get that $a, c \in A$ and thus $\operatorname{proj}(R)$ and $T^{\prime}$ are not chord separated, which finishes the proof of the claim.

Our situation looks as follows. We have a maximal by inclusion chord separated collection $\mathcal{D}^{\prime} \subset 2^{[r]}$. It clearly contains all the cyclic intervals, including all the one-element sets. In order to complete the proof of Lemma 4.8, we need to show that $\mathcal{D}^{\prime}$ contains at least one non-trivial two-element set, where non-trivial means that it is not a cyclic interval.

For a set $T \subset[r]$, define

$$
\operatorname{maxgap}(T):=\max _{(a, b) \cap T=\emptyset}|(a, b)| .
$$


In other words, maxgap $(T)$ is the maximal size of the distance between two consecutive entries of $T$. Choose a set $T \in \mathcal{D}^{\prime}$ satisfying:

- $T$ is non-trivial;

- there is no other non-trivial $T^{\prime} \in \mathcal{D}^{\prime}$ with $\operatorname{maxgap}\left(T^{\prime}\right)>\operatorname{maxgap}(T)$.

Let $(a, b)$ be an interval that maximizes the right hand side of (3). Then $a, b \in T$. Since $T$ is non-trivial, it is not the case that $T=[b, a]$ and thus there is an element $c \in(b, a)-T$. Consider all pairs $\left(T^{\prime}, c^{\prime}\right)$ satisying

- $T^{\prime} \in \mathcal{D}^{\prime}$

- $a, b \in T^{\prime}$

- $(a, b) \cap T^{\prime}=\emptyset$;

- $c^{\prime} \in(b, a)-T^{\prime}$.

Among all such pairs $\left(T^{\prime}, c^{\prime}\right)$, choose the one which minimizes the size of $\left(b, c^{\prime}\right)$. Thus $\left[b, c^{\prime}\right) \subset T^{\prime}$ but $c^{\prime} \notin T^{\prime}$. Let $d \in T^{\prime}$ be such that $\left(c^{\prime}, d\right) \cap T^{\prime}=\emptyset$. Consider the set $\left\{d, c^{\prime}-1\right\}$. Certainly this is a nontrivial subset of $[r]$, because $\left(c^{\prime}-1, d\right)$ contains $c^{\prime}$ while $\left(d, c^{\prime}-1\right)$ contains either $a$ or $b$. Thus if $\left\{d, c^{\prime}-1\right\} \in \mathcal{D}^{\prime}$ then we are done. Assume that this is not the case, then there is a set $R \subset[r]$ such that $d, c^{\prime}-1 \notin R$ and $e, f \in R$ with $e \in\left(c^{\prime}-1, d\right)$ and $f \in\left(d, c^{\prime}-1\right)$. If $f \notin T^{\prime}$ then $R$ and $T^{\prime}$ are not chord separated. Therefore $(a, b)$ contains no such $f$ 's and thus $(a, b) \cap R$ has to be empty. If either $a \notin R$ or $b \notin R$ then $\operatorname{maxgap}(R)>\operatorname{maxgap}(T)$ which is impossible since $R$ is non-trivial. Thus $a, b \in R$. But since $c^{\prime}-1 \notin R$, we get a contradiction with the fact that the size of $\left(b, c^{\prime}\right)$ was minimal possible. Thus $\left\{d, c^{\prime}-1\right\} \in \mathcal{D}^{\prime}$ and we have found a non-trivial two element subset in $\mathcal{D}^{\prime}$. It means that $\mathcal{D}$ contains the set $S a_{d} a_{c^{\prime}-1}$ as well as $S, S a_{d}, S a_{c^{\prime}-1}$ which means that we have an edge that subdivides the white polygon $P$ into two smaller polygons. This finishes the proof of Lemma 4.8.

We have proven both lemmas and therefore we are done with Proposition 4.6 as well as Theorem 1.2 .

\section{REFERENCES}

[1] Nima Arkani-Hamed, Jacob L. Bourjaily, Freddy Cachazo, Alexander B. Goncharov, Alexander Postnikov, and Jaroslav Trnka. Scattering amplitudes and the positive grassmannian. arXiv:1212.5605, 2012.

[2] L. J. Billera, M. M. Kapranov, and B. Sturmfels. Cellular strings on polytopes. Proc. Amer. Math. Soc., 122(2):549-555, 1994. 
[3] Anders Björner, Michel Las Vergnas, Bernd Sturmfels, Neil White, and Günter M. Ziegler. Oriented matroids, volume 46 of Encyclopedia of Mathematics and its Applications. Cambridge University Press, Cambridge, second edition, 1999.

[4] Jochen Bohne. Eine kombinatorische analyse zonotopaler raumaufteilungen. Univ., Diss.-Bielefeld, 1992.

[5] Vladimir I. Danilov, Alexander V. Karzanov, and Gleb A. Koshevoy. On maximal weakly separated set-systems. J. Algebraic Combin., 32(4):497-531, 2010.

[6] Vladimir I Danilov, Alexander V Karzanov, and Gleb A Koshevoy. Combined tilings and the purity phenomenon on separated set-systems. arXiv preprint arXiv:1401.6418, 2014.

[7] Yuji Kodama and Lauren Williams. KP solitons and total positivity for the Grassmannian. Invent. Math., 198(3):637-699, 2014.

[8] Yuji Kodama and Lauren K. Williams. KP solitons, total positivity, and cluster algebras. Proc. Natl. Acad. Sci. USA, 108(22):8984-8989, 2011.

[9] Bernard Leclerc and Andrei Zelevinsky. Quasicommuting families of quantum Plücker coordinates. In Kirillov's seminar on representation theory, volume 181 of Amer. Math. Soc. Transl. Ser. 2, pages 85-108. Amer. Math. Soc., Providence, RI, 1998.

[10] Suho Oh, Alexander Postnikov, and David E. Speyer. Weak separation and plabic graphs. Proc. Lond. Math. Soc. (3), 110(3):721-754, 2015.

[11] Alexander Postnikov. Total positivity, Grassmannians, and networks. arXiv preprint math/0609764, 2006.

[12] Victor Reiner. The generalized Baues problem. In New perspectives in algebraic combinatorics (Berkeley, CA, 1996-97), volume 38 of Math. Sci. Res. Inst. Publ., pages 293-336. Cambridge Univ. Press, Cambridge, 1999.

[13] Joshua S. Scott. Quasi-commuting families of quantum minors. J. Algebra, 290(1):204-220, 2005.

[14] Joshua S. Scott. Grassmannians and cluster algebras. Proc. London Math. Soc. (3), 92(2):345-380, 2006.

[15] Günter M. Ziegler. Higher Bruhat orders and cyclic hyperplane arrangements. Topology, 32(2):259-279, 1993.

E-mail address: galashin@mit.edu

Department of Mathematics, Massachusetts Institute of Technology, CAmbridge, MA, 02139, USA. 